\title{
A note on nil-clean rings
}

\author{
Peter V. Danchev \\ Institute of Mathematics and Informatics, \\ Bulgarian Academy of Sciences, \\ "Acad. G. Bonchev" str., bl. 8, 1113 Sofia, Bulgaria \\ email: danchev@math.bas.bg; \\ pvdanchev@yahoo.com
}

\begin{abstract}
We study a special kind of nil-clean rings, namely those nil-clean rings whose nilpotent elements are difference of two "left-right symmetric" idempotents, and prove that in some various cases they are strongly $\pi$-regular. We also show that all nil-clean rings having cyclic unit 2-groups are themselves strongly nil-clean of characteristic 2 (and thus they are again strongly $\pi$-regular).
\end{abstract}

\section{Introduction and background}

Everywhere in the text of the present paper, all our rings $R$ are assumed to be associative, containing the identity element 1 , which in general differs from the zero element 0 of $R$. Our terminology and notations are mainly standard being in agreement with [9]. Exactly, $U(R)$ denotes the set of all units in $R$, $\operatorname{Id}(R)$ the set of all idempotents in $R, \operatorname{Nil}(R)$ the set of all nilpotents in $R$ and $J(R)$ the Jacobson radical of $R$.

A ring $\mathrm{R}$ is called von Neumann regular or just regular for short if, for any element $r \in R$, there is an element $a \in R$ such that $r=$ rar. In the case when $a=1$, we have that $r=r^{2}$ and these rings are known to be boolean. Generalizing regularity, a ring $R$ is called $\pi$-regular if, for each $r \in R$, there are $i \in \mathbb{N}$ and $b \in R$ both depending on $r$ such that $r^{i}=r^{i} b r^{i}$. Likewise, a ring $R$ is called strongly $\pi$-regular if, for every $r \in R$, there exist $j \in \mathbb{N}$ and 
$c \in R$ both depending on $r$ with $r^{j}=r^{j+1} c$. It is well known that strongly $\pi$-regularity implies $\pi$-regularity, while the converse is wrong as some critical examples show (see, e.g., [9]).

On the other hand, referring to [7] for a more account, we shall say that a ring is nil-clean provided each its element is a sum of a nilpotent and an idempotent. If these two elements commute, the nil-clean ring is said to be strongly nil-clean. While nil-clean rings are not completely characterized up to an isomorphism yet, this was successfully done in [4] by proving that a ring $R$ is strongly nil-clean if, and only if, the quotient ring $R / J(R)$ is boolean and $J(R)$ is nil.

That is why, classifying the structure of some special types of nil-clean rings will be of some interest and importance. Our workable purpose here is to examine those nil-clean rings whose nilpotents are differences of two (special) idempotents. Specifically, we shall prove that in Theorem 1 presented below that every nil-clean ring having only nilpotents which are difference of two special (so-called "left-right symmetric") idempotents is strongly $\pi$-regular. This contrasts an example due to Šter in [11] who constructed a nil-clean ring of unbounded index of nilpotence which is not strongly $\pi$-regular. Note that by an appeal to [6, Corollary 3.12] nil-clean rings of bounded index of nilpotence are always strongly $\pi$-regular. We also consider the challenging question of when a nil-clean ring with finite (in particular, cyclic) unit group is strongly nil-clean. It is necessarily such a group to be consisting only of elements of order being a power of 2 , and the ring will be of characteristic 2 too.

\section{Main results}

We separate our chief results into two subsections as follows:

\subsection{Nil-clean rings with nilpotents as a sum of two special idempotents}

We start our assertions with the next one.

Proposition 1 If $\mathrm{R}$ is a nil-clean ring such that each nilpotent is a difference of two commuting idempotents, then $\mathrm{R}$ is a boolean ring.

Proof. We first claim that such a ring $R$ is of characteristic 2. Indeed, as $2 \in \operatorname{Nil}(R)$ (see, e.g., [7]), one writes that $2=e-f$ for some $e, f \in \operatorname{Id}(R)$. Hence, it easily follows that $e f=f e$ even not assuming this a priory and, 
therefore, $2^{3}=(e-f)^{3}=e-f=2$. This means that $6=0$, i.e., $2=0$ because $3 \in \mathrm{U}(\mathrm{R})$ and the claim is sustained.

Moreover, we assert that $\mathrm{R}$ has to be abelian, that is, all its idempotents are central. In fact, given an arbitrary $a \in R$ and an arbitrary $e \in \operatorname{Id}(R)$, one sees that $e a(1-e) \in \operatorname{Nil}(R)$ and thus $e a(1-e)=e_{1}+e_{2}$ for some $e_{1}, e_{2} \in \operatorname{Id}(R)$ with $e_{1} e_{2}=e_{2} e_{1}$. Squaring this, it follows at once that $0=e_{1}+e_{2}$ since $2=0$ which yields $e a=e a e$. Similarly, one derives that ae =eae by looking at the element $(1-e) a e$, which allows us to conclude that ae $=e a$, as asserted.

We next arrive at the fact that $R$ is semi-primitive, which is equivalent to $J(R)=\{0\}$. To verify this, given any element $z \in J(R)$, one may write that $z=$ $e-f$ for some $e, f \in \operatorname{Id}(R)$ with $e f=f e$ since $J(R)$ is nil (see, for instance, [7]). Now, taking into account that $2=0$, we find that $z^{2}=z$ whence $z(z-1)=0$ ensuring that $z=0$ because $z-1 \in \mathrm{U}(\mathrm{R})$. Thus $\mathrm{R}$ is semi-primitive, as claimed.

Furthermore, we may apply either [4] or [7] to get the desired boolean property of R.

It was established in [8, Proposition 1] that any nilpotent matrix over a field is a difference of two idempotent matrices (for another approach see [10] as well). This major statement allows us to extract the following assertion, independently proved also in [10] and partially in [3].

Lemma 1 In regular rings all nilpotent elements are difference of two idempotents.

Proof. Consulting with the main result from [1] which shows that, in an arbitrary ring, a nilpotent with all powers regular can be thought of as locally just a nilpotent matrix in Jordan or Weyr form. With this at hand, the aforementioned matrix result in [8] gives the desired presentation.

Imitating [3], two idempotents e,f are called left-right symmetric if the two equalities $e f=e$ and $f e=f$ hold. It is evident that both $e$ and $f$ are somewhat "left-active" in the sense that they are "preserved on the left multiplication".

So, we have accumulated all the information necessary to establish the following.

Theorem 1 Every nil-clean ring in which all nilpotents are difference of two left-right symmetric idempotents are strongly $\pi$-regular.

Proof. We foremost assert that for such a ring $R$ it must be that $\operatorname{char}(R)=2$. To see that, as $2 \in \operatorname{Nil}(R)$ holds in view of [7], one writes that $2=e_{1}-e_{2}$ for two $e_{1}, e_{2} \in \operatorname{Id}(R)$. This surely means that $e_{1}$ and $e_{2}$ do commute, so that 
$2^{3}=\left(e_{1}-e_{2}\right)^{3}=e_{1}-e_{2}=2$ whence $6=0$. Consequently, $2=0$ because $3 \in \mathrm{U}(\mathrm{R})$, as asserted.

For such a ring $R$, given an arbitrary $q \in \operatorname{Nil}(R)$, we write that $q=e-f=$ $e+f$ for some two $e, f \in \operatorname{Id}(R)$ with $e f=e$ and $f e=f$. We, therefore, obtain by squaring that $q^{2}=2 q=0$. Thus $R$ is of bounded index of nilpotence and [6, Corollary 3.12] is a guarantor for the validity of our assertion that $R$ is strongly $\pi$-regular.

The given proof allows us to consider whether a more general situation in which we have slightly amended relationships between $e$ and $\mathrm{f}$, that are, efe $=$ $e$ and $f e f=f$. Certainly, $e f=e$ forces $e f e=e$ as well as $f e=f$ forces $f e f=f$. Furthermore, writing $q=e+f$ and squaring this, we deduce that $q^{2}-q=e f+f e$. Again squaring the last equality, we derive that $q^{4}+q^{2}=$ $\left(q^{2}-q\right)^{2}=e f e f+e f e+f e f+f e f e=e f+e+f+f e=q^{2}$. Finally, $q^{4}=0$ and hence $\mathrm{R}$ is with bounded index of nilpotence, too.

We can now mention some constructions of nil-clean rings having only nilpotent elements which are difference of two idempotents.

Remark 1 By what we have just previously shown, a crucial example of such a sort of nil-clean rings is any nil-clean ring which is simultaneously regular - in fact, such is, for instance, the ring $\mathbb{M}_{\mathfrak{n}}\left(\mathbb{Z}_{2}\right)$ for all $\mathrm{n} \geq 1$ by an appeal to [2] and to the well-known fact from [9] that it is a regular ring because so is $\mathbb{Z}_{2}$. Indeed, this is not always possible as it was recently exhibited in [11] an ingenious example of a nil-clean ring of characteristic 2 which is not strongly $\pi$-regular as well as of a nil-clean ring of characteristic 4 which is not $\pi$-regular.

An other interesting example of a nil-clean ring whose nilpotent elements are differences of two idempotents and which ring is not regular (due to the fact that it has a non-zero Jacobson radical) is the upper triangular matrix ring $\mathbb{T}_{2}\left(\mathbb{Z}_{2}\right)$, which fact we leave to the interested reader for a direct inspection. This ring is, however, strongly $\pi$-regular.

Moreover, the indecomposable nil-clean ring $\mathbb{Z}_{4}$ does not have the indicated above specific property of its nilpotents since $2 \neq 0$ in it.

We end our work in this subsection with the following challenging problem.

Problem 1 Characterize nil-clean rings whose nilpotent elements are differences of two arbitrary idempotents. 


\subsection{Nil-clean rings with cyclic unit group}

In $[5, \mathrm{p} .81]$ it was asked of whether or not a clean ring with cyclic units is strongly clean. We shall resolve this question in the case of nil-clean rings (note that nil-clean rings are always clean and a clean ring is the one whose elements are sums of a unit and an idempotent; if these two elements commute, the clean ring is called strongly clean). It was established in [4, Corollary 4.10] that a nil-clean is strongly nil-clean if, and only if, its unit group is a 2-group.

We are now arriving at the following statement.

Theorem 2 Suppose $\mathrm{R}$ is a nil-clean ring with cyclic $\mathrm{U}(\mathrm{R})$. Then $\mathrm{R}$ is strongly nil-clean of characteristic 2 if, and only if, $\mathrm{U}(\mathrm{R})$ is a 2-group.

Proof. If we assume for a moment that $U(R)=\{1\}$, then $\operatorname{Nil}(R)=\{0\}$ as $1+\operatorname{Nil}(R) \subseteq U(R)$, so that $R$ must be boolean whence strongly nil-clean. So, we shall assume hereafter that $\mathrm{U}(\mathrm{R}) \neq\{1\}$.

Firstly, to prove the "right-to-left" implication, assume that $\mathrm{U}(\mathrm{R})$ is a cyclic 2 -group. Thus, as commented above, it follows immediately from [4, Corollary 4.10] that $\mathrm{R}$ is strongly nil-clean. What remain to show is that $2=0$ holds in $\mathrm{R}$. Indeed, since $2 \in \operatorname{Nil}(R)$, one observes that the infinite sequence $\{3,5,7, \ldots, 2 k-$ $1,2 k+1, \ldots\}$ will invert in $R$ for any $k \in \mathbb{N}$. But as $U(R)$ is finite, there will exist a natural number $k$ with $2 k-1=2 k+1$, so that $2=0$ is really fulfilled.

Secondly, the direct application of [4, Corollary 4.10] gives the "left-to-right" part, as desired.

We finish our work in this subsection with the following useful comments which shed some further light on the explored theme.

Remark 2 For nil-clean rings with finite unit group the above theorem is not longer true: in fact, as an example we can consider the $2 \times 2$ matrix ring $\mathbb{M}_{2}\left(\mathbb{Z}_{2}\right)$ which, in accordance with [2], is nil-clean but surely not strongly nil-clean (however, it is strongly $\pi$-regular being finite). This suggests to extract even the more general claim that nil-clean rings with finite unit group are strongly $\pi$-regular of characteristic 2. In fact, as unipotents (= the sum of 1 and a nilpotent) are always units, it readily follows that the set of nilpotents is also finite and so the ring is with bounded index of nilpotence. We, therefore, can apply [6, Corollary 3.12] to get the wanted claim. That char $(\mathrm{R})=2$ follows now in the same manner as in the proof of Theorem 2.

In closing, we pose a few intriguing problems of some interest and importance which immediately arise. 
Problem 1. If $R$ is a nil-clean ring with bounded $U(R)$, does it follow that $R$ is (strongly) $\pi$-regular?

Problem 2. If $R$ is a nil-clean ring of characteristic 2 and $U(R)$ is a $p$-group (or, respectively, a $2 p$-group) for some prime $p$, is it true that $R$ is (strongly) $\pi$-regular?

For eventual counterexamples in case we have dropped some of the requirements, see Examples 3.1 and 3.2 from [11].

In regard to both sections explored above, one may state the following:

Problem 3. Is any nil-clean ring $R$ such that its nilpotents are differences of two idempotents always $\pi$-regular? In particular, if $J(R)=0$, is then $R$ necessarily von Neumann regular.

In fact, each such nil-clean ring is of characteristic 2. If the above question holds in the affirmative, this will be in sharp contrast to the recent example by Šter from [11] showing that there is a nil-clean ring which is not $\pi$-regular.

Letting $Q \operatorname{Nil}(R)$ be the set of all quasi-nilpotent elements of the ring $R$, we note that both inclusions $\operatorname{Nil}(\mathrm{R}) \subseteq \mathrm{QNil}(\mathrm{R})$ and $\mathrm{J}(\mathrm{R}) \subseteq \mathrm{QNil}(\mathrm{R})$ hold. We thereby come in mind to our next question as follows:

Problem 4. Examine those (nil-clean) rings for which the equality $U(R)=$ $1+\mathrm{QNil}(\mathrm{R})$ is true.

Notice that the condition $\mathrm{U}(\mathrm{R})=1+\operatorname{Nil}(\mathrm{R})+\mathrm{J}(\mathrm{R})$ obviously implies the condition $U(R)=1+Q N i l(R)$, as in the latter situation we shall say that the ring $R$ has quasi-nilpotent units.

\section{Acknowledgement}

The author is grateful to the referee for his/her careful reading of the manuscript and the competent comments and suggestions made as well as he is obliged to the chief-editor, Prof. Robert Szasz, for the professional editorial management of the current submission.

Funding: The article is partially supported by the Bulgarian National Science Fund under Grant KP-06 No 32/1 of December 07, 2019 and by project РД08-136/04.02.2020 of the University of Shumen "Konstantin Preslavsky". 


\section{References}

[1] K. I. Beidar, K. C. O’Meara and R. M. Raphael, On uniform diagonalisation of matrices over regular rings and one-accesible regular algebras, Commun. Algebra, 32 (2004), 3543-3562.

[2] S. Breaz, G. Călugăreanu, P. Danchev and T. Micu, Nil-clean matrix rings, Linear Algebra and Appl., (10) 439 (2013), 3115-3119.

[3] P. V. Danchev, Weakly exchange rings whose units are sums of two idempotents, Vestnik St. Petersburg Univ., Math., Mech. \& Astronomy, (2) 6 (64) (2019), 265-269.

[4] P. V. Danchev and T. Y. Lam, Rings with unipotent units, Publ. Math. Debrecen, (3-4) 88 (2016), 449-466.

[5] P. Danchev and J. Matczuk, n-Torsion clean rings, Contemp. Math., 727 (2019), 71-82.

[6] P. Danchev and J. Šter, Generalizing $\pi$-regular rings, Taiwanese J. Math., (6) 19 (2015), 1577-1592.

[7] A. J. Diesl, Nil clean rings, J. Algebra, 383 (2013), 197-211.

[8] R. E. Hartwig and M. S. Putcha, When is a matrix a difference of two idempotents, Linear and Multilinear Algebra, (4) 26 (1990), 267-277.

[9] T.-Y. Lam, A First Course in Noncommutative Rings, Second Edition, Graduate Texts in Math., Vol. 131, Springer-Verlag, Berlin-HeidelbergNew York, 2001.

[10] K. C. O'Meara, Nilpotents often the difference of two idempotents, private correspondence on draft privately circulated on March 2018.

[11] J. Šter, On expressing matrices over $\mathbb{Z}_{2}$ as the sum of an idempotent and a nilpotent, Linear Algebra Appl., 544 (2018), 339-349.

Received: December 17, 2019 